\title{
Orangutan Alu quiescence reveals possible source element: support for ancient backseat drivers
}

\author{
Jerilyn A Walker ${ }^{1 \dagger}$, Miriam K Konkel ${ }^{1 \dagger}$, Brygg Ullmer ${ }^{2}$, Christopher P Monceaux ${ }^{1,3,4}$, Oliver A Ryder ${ }^{5}$, Robert Hubley ${ }^{6}$, \\ Arian FA Smit ${ }^{6}$ and Mark A Batzer ${ }^{1 *}$
}

\begin{abstract}
Background: Sequence analysis of the orangutan genome revealed that recent proliferative activity of Alu elements has been uncharacteristically quiescent in the Pongo (orangutan) lineage, compared with all previously studied primate genomes. With relatively few young polymorphic insertions, the genomic landscape of the orangutan seemed like the ideal place to search for a driver, or source element, of Alu retrotransposition.

Results: Here we report the identification of a nearly pristine insertion possessing all the known putative hallmarks of a retrotranspositionally competent Alu element. It is located in an intronic sequence of the DGKB gene on chromosome 7 and is highly conserved in Hominidae (the great apes), but absent from Hylobatidae (gibbon and siamang). We provide evidence for the evolution of a lineage-specific subfamily of this shared Alu insertion in orangutans and possibly the lineage leading to humans. In the orangutan genome, this insertion contains three orangutan-specific diagnostic mutations which are characteristic of the youngest polymorphic Alu subfamily, AluYe5b5_Pongo. In the Homininae lineage (human, chimpanzee and gorilla), this insertion has acquired three different mutations which are also found in a single human-specific Alu insertion.
\end{abstract}

Conclusions: This seemingly stealth-like amplification, ongoing at a very low rate over millions of years of evolution, suggests that this shared insertion may represent an ancient backseat driver of Alu element expansion.

\section{Background}

The amplification of Alu elements has been ongoing in primate genomes for about 65 million years [1,2]. They typically mobilize via a 'copy and paste' mechanism through an RNA intermediate, a process termed targetprimed reverse transcription (TPRT) [3]. Alu elements are non-autonomous and utilize the enzymatic machinery of autonomous LINE elements (L1) to mobilize $[1,4,5]$. Due to the staggered DNA cuts of the genome by the L1-derived endonuclease during TPRT, Alu insertions are flanked by short sequences of duplicated host DNA called target site duplications (TSDs), which can be used to identify the insertion event. Alu elements accumulate in an 'identical by descent' manner. This

\footnotetext{
* Correspondence: mbatzer@lsu.edu

† Contributed equally

'Department of Biological Sciences, Louisiana State University, 202 Life

Sciences Building, Baton Rouge, LA 70803, USA

Full list of author information is available at the end of the article
}

means that the ancestral state at any locus is the absence of the element and, conversely, that the presence of a shared element with matching TSDs at a given locus indicates a common ancestor. Thus, Alu elements are considered essentially homoplasy-free characters $[1,6]$. Although the autonomous features of L1 are straightforward, the identification of Alu element insertions that retain the ability to propagate copies of themselves has remained somewhat elusive. This is primarily because Alu elements do not contain coding sequence and the vast majority of insertions are highly similar to each other. Structural factors, such as having an intact promoter region, low sequence diversity from a known polymorphic subfamily, close proximity of the polymerase III (Pol III) termination signal to the end of the element and the length of the poly(A) tail, have all been associated with $A l u$ retrotransposition ability $[4,7,8]$. Yet, to date, only one Alu source element has been identified in humans with clear evidence that it produced an

\section{Biomed Central}


offspring element [4]. This rare finding is due in part to the large landscape of hundreds of relatively young elements with limited knowledge about what characteristics make them retrotransposition competent. In the case of the orangutan, the landscape of relatively young elements is quite sparse [9].

Orangutans are characterized by a relatively long lifespan among primates (35 to 45 years in the wild) combined with the longest average inter-birth interval between offspring (8 years) $[9,10]$. These relatively low reproduction rates along with their relatively large body size compared to other great apes are consistent with a 'slow' life history strategy, impacting their genomic architecture over time. Investigation of the orangutan draft genome sequence [ponAbe2] revealed a very low retrotransposition rate of $A l u$ elements in the orangutan lineage leading to Pongo abelii (Sumatran orangutan), while seeming to maintain an L1 activity comparable to other primates [9]. This finding was in particular startling because all primate genomes studied to date showed evidence of strong ongoing Alu and L1 retrotransposition [11-13]. Variation in Alu retrotransposition activity within different primate species has been reported previously [14] and is known to vary over the course of evolution [15], but the orangutan genome provided the first evidence of such a dramatic decline in $A l u$ retrotransposition in primates. An extensive analysis of the ponAbe 2 assembly identified only approximately 250 lineage-specific Alu insertions, which translates to an average of only about 18 new insertions per million years [9]. This is in sharp contrast to analyses of the human and chimpanzee genomes, in which approximately 5,000 and 2,300 lineagespecific $A l u$ insertions were identified, respectively $[9,11,13]$. Of the orangutan-specific Alu subfamilies, three were determined to be the youngest. We have termed these AluYc1a5_Pongo, AluYe5a2_Pongo and AluYe5b5_Pongo based on sequence comparisons to previously identified human Alu subfamilies and using the standardized nomenclature for $A l u$ repeats [16] (see methods for naming convention). From these three youngest orangutan $A l u$ subfamilies, the 44 youngest appearing elements, on the basis of divergence from their subfamily consensus sequences, were analyzed for insertion presence or absence in a DNA panel of orangutans and other primates for the population genetics portion of the Orangutan Genome Project [9] (supplementary section 19); and only 13 were shown to be polymorphic in the orangutans evaluated. We postulated that the low number of recent $A l u$ insertions representing only three young subfamilies might provide an ideal genomic landscape to search for their source elements.

\section{Results}

We used BLAT [17] to computationally search the orangutan ponAbe2 genome assembly for the matches with the highest homology to each of the three polymorphic subfamily consensus sequences. The vast majority of the closest matches were young appearing (low divergence from their respective consensus sequences) loci already evaluated by PCR in the previous study [9]. However, the results for the closest matches to the AluYe5b5_Pongo consensus sequence revealed a previously uncharacterized $A l u$ insertion on chromosome 7 (Chr7) with > 99\% sequence identity to the consensus sequence (Table 1). Using the University of California Santa Cruz (UCSC) Genome Browser [18,19], it became evident that this insertion was not a recent event overlooked by our previous analyses, but was shared by the human (hg18) and chimpanzee (panTro2) genomes, while absent from the rhesus macaque genome (rheMac2). Therefore, this locus had been computationally filtered from our previous study of young orangutan-specific Alu insertions because it was also present in the human genome.

The Genome Browser function [18,19] indicated that this shared Chr7 Alu insertion is located in an intronic sequence of the $D G K B$ gene (coding for diacylglycerol kinase beta enzyme) [20]. The $D G K B$ gene is one of several mammalian genes that encode for diacylglycerol kinases. Diacylglycerol kinases are involved in cellular processes by regulating diacylglycerol levels. For $D G K B$, two alternatively spliced transcript variants have been identified [21]. PCR evidence confirmed the presence of this Chr7 Alu insertion in human, chimpanzee, gorilla and orangutan, and indicated the absence from siamang, gibbon, African green monkey and rhesus macaque (Figure 1), suggesting this element is specific to Hominidae (the great apes) and absent from Hylobatidae (the gibbons and siamang) and other extant primates. This dates the insertion event to at least 16 million years ago using species divergence estimates from Locke et al. [9].

An alignment of the BLAT sequence from human (hg18), chimpanzee (panTro2) and orangutan (ponAbe2) revealed a remarkably conserved left monomer and few nucleotide substitutions in the right monomer in all three species (Figure 2 and Additional file 1: Figure S1). In order to verify the PCR data of a shared insertion, DNA from eight different orangutans (four Sumatran and four Bornean), a lowland gorilla and two pygmy chimpanzees (bonobo) were PCR amplified at this locus. PCR products were gel purified, cloned and Sanger sequenced to confirm that the homologous insertions shared matching TSDs and flanking sequence. A sequence alignment of these results is shown in Additional file 1: Figure S1 and a list of the DNA samples 
Table 1 Orangutan BLAT [ponAbe2] results for Alu subfamily AluYe5b5_Pongo

\begin{tabular}{llllll}
\hline Identity (\%) & Chromosome & Start & End & Distribution & Frequency \\
\hline 99.70 & 12 & 90005006 & 90005290 & Poly Sum/Bornean & 0.5405 \\
\hline 99.30 & 7 & 70375547 & 70375831 & Shared H/C/G/O & 1.0000 \\
\hline 98.60 & 17 & 56932716 & 56932999 & Poly Sum specific & 0.0811 \\
\hline 99.30 & 13 & 109637257 & 109637541 & Poly Sum specific & 0.2027 \\
\hline 98.30 & 21 & 23655335 & 23655618 & Poly Sum specific & 0.0946 \\
\hline 97.90 & $2 \mathrm{~b}$ & 3809376 & 3809659 & Poly Sum specific & 0.3513 \\
\hline
\end{tabular}

H/C/G/O: human, chimpanzee, gorilla, orangutan; Poly: polymorphic; Sum: Sumatran.

used for sequencing is shown in Additional file 1: Table S1. The left monomer of the Alu element is highly conserved, especially among the orangutans, and all species share the TTTT Pol III transcription termination signal in the 3' TSD. These sequencing results confirmed that the Chr7 Alu element was shared among all great ape species, that the sequence in all individual orangutans was nearly identical to the AluYe5b5_Pongo consensus sequence and that it possessed some traditional hallmarks associated with retrotransposition ability.
Members of the orangutan AluYe5b5_Pongo subfamily most closely matching the $\mathrm{Chr} 7 \mathrm{Alu}$ consensus sequence from the ponAbe 2 genome assembly ( $\mathrm{n}=7$ loci) were evaluated by PCR for presence or absence and allele frequency distribution (Table 2) in a DNA panel including 37 orangutans (see Methods and Additional file 2: Genotypes/DNA samples). Next, we computationally searched the chimpanzee genome (panTro2) and human genome (hg18) for the closest matches to their respective Chr7 Alu consensus sequences using BLAT. BLAT

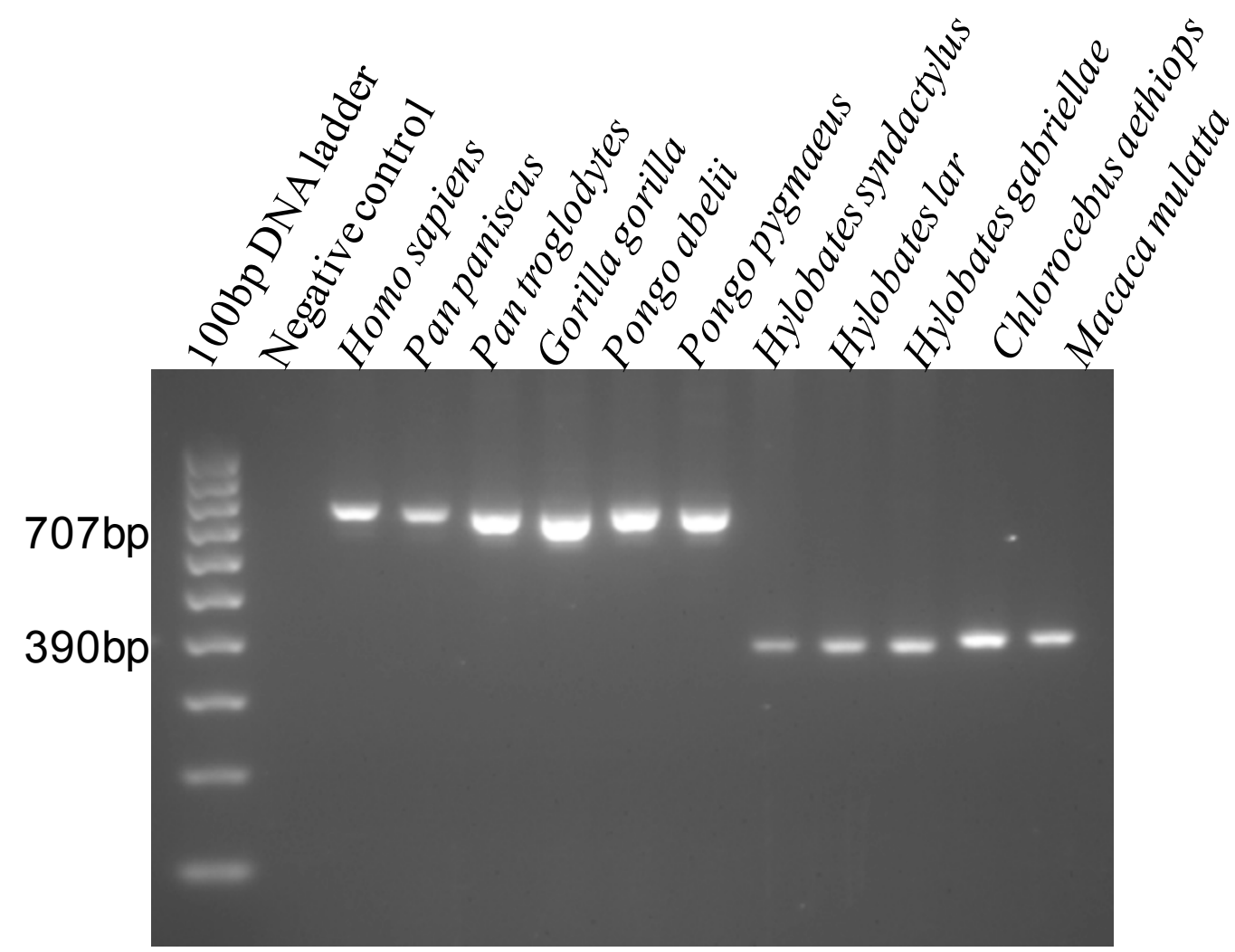

Figure 1 Analysis of the chromosome 7 shared Alu insertion using polymerase chain reaction. The Alu insertion is shared among Hominidae (the great apes) and absent from other extant primates. The filled site is approximately $707 \mathrm{bp}$ (lanes 3 to 8 ) and the empty site is 390 bp (lanes 9 to 13). Lanes: (1) 100 bp DNA ladder; (2) negative control; (3) human; (4) bonobo chimpanzee; (5) common chimpanzee; (6) lowland gorilla; (7) Sumatran orangutan; (8) Bornean orangutan; (9) siamang; (10) white-handed gibbon; (11) red-cheeked gibbon; (12) African green monkey; (13) rhesus macaque. 


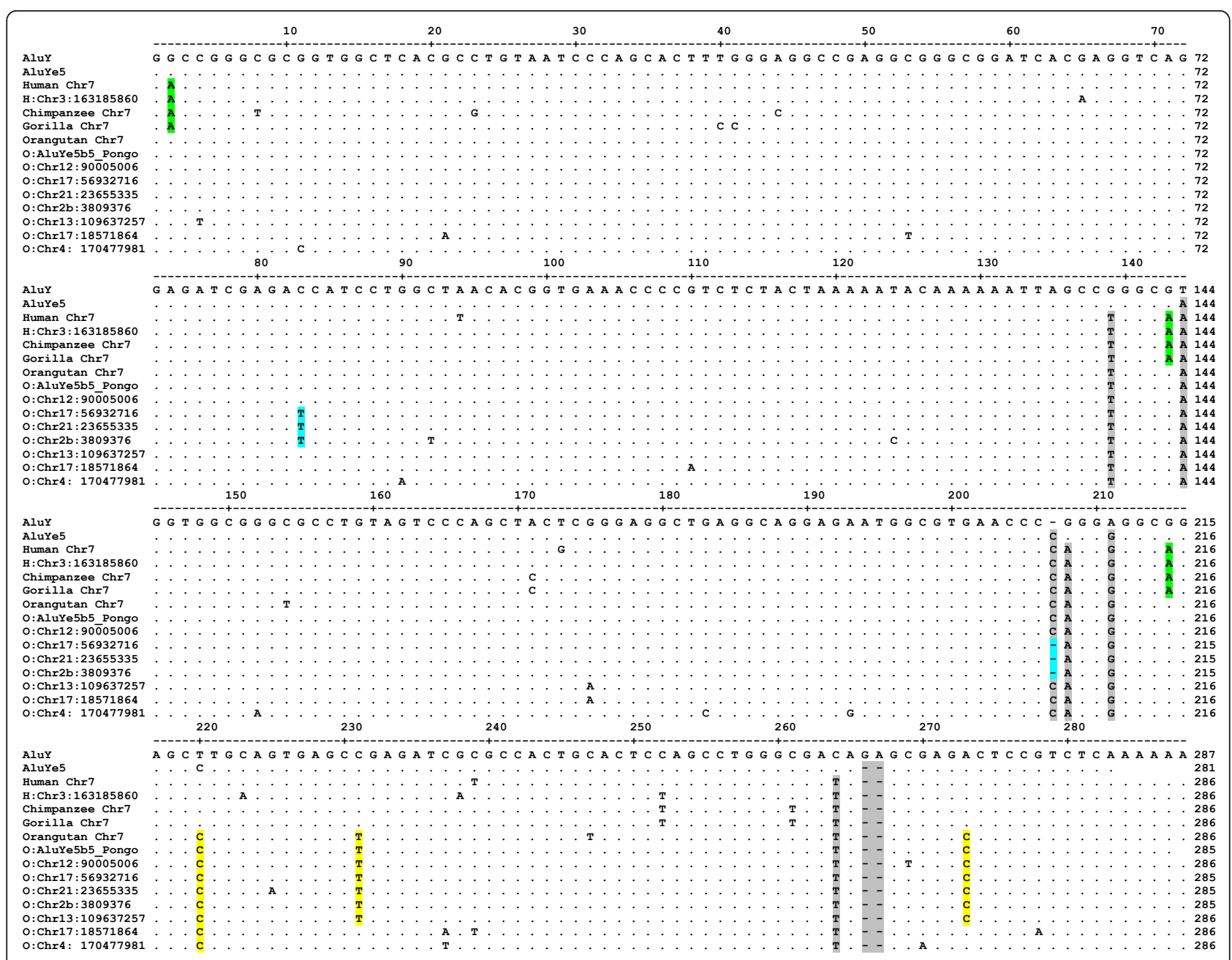

Figure 2 Alu Sequence alignment. The consensus sequence for the ancestral AluY subfamily is shown at the top. The dots represent the same nucleotide as AluY. Deletions are shown as dashes and mutations are shown as the corrected base. The chromosome 7 locus has a number of mutations different from AluY that are shared by all investigated species (highlighted in gray) and are all located in the right monomer of the element following the middle A-rich region. Post-insertion, the chromosome 7 locus in the orangutan (labeled Orangutan Chr7) independently acquired sequential diagnostic mutations (highlighted in yellow) shared by all polymorphic loci of the young AluYe5b5_Pongo subfamily in orangutans (starting with $\mathrm{O}: \mathrm{Chr}$ ). At some point, one of the AluYe5b5_Pongo members subsequently acquired one substitution and one deletion (highlighted in aqua) and has propagated as a daughter subfamily. Following the divergence of orangutan and the lineage leading to humans, the chromosome 7 locus acquired three substitutions (highlighted in green) shared in gorilla, chimpanzee and human. There is one humanspecific Alu insertion, $\mathrm{H}$ : Chr3, which shares these three variants.

Table 2 Orangutan BLAT [ponAbe2] results for orangutan chromosome 7 locus

\begin{tabular}{llllll}
\hline Identity (\%) & Chromosome & Start & End & Distribution & Frequency \\
\hline 100.00 & 7 & 70375547 & 70375836 & Shared H/C/G/O & 1.0000 \\
\hline 99.00 & 12 & 90005006 & 90005295 & Poly Sum/Bornean & 0.5405 \\
\hline 98.00 & 17 & 56932716 & 56933004 & Poly Sum specific & 0.0811 \\
\hline 98.70 & 13 & 109637257 & 109637546 & Poly Sum specific & 0.2027 \\
\hline 97.60 & 21 & 23655335 & 23655623 & Poly Sum specific & 0.0946 \\
\hline 97.30 & $2 \mathrm{~b}$ & 3809376 & 3809664 & Poly Sum specific & 0.3513 \\
\hline 96.30 & 17 & 18571864 & 18572153 & Orangutan specific & fixed \\
\hline 95.90 & 4 & 170477976 & 170478265 & Orangutan specific & fixed \\
\hline
\end{tabular}

H/C/G/O: human, chimpanzee, gorilla, orangutan; Poly: polymorphic; Sum: Sumatran. 
Table 3 Human BLAT [hg18] results for human chromosome 7 locus

\begin{tabular}{llllll}
\hline Identity (\%) & Chromosome & Start & End & Distribution & Frequency \\
\hline 100.00 & 7 & 14399134 & 14399438 & Shared H/C/G/O & 1.0000 \\
\hline 97.10 & 3 & 163185859 & 163186163 & Human specific & fixed \\
\hline
\end{tabular}

H/C/G/O: human, chimpanzee, gorilla, orangutan.

results for the human genome identified one Alu insertion on chromosome 3 that was $>97 \%$ identical (Table 3 ) and shared the same three substitutions, suggesting that it may have derived from the Chr7 source locus. PCR primers were designed for the chromosome 3 locus and genotyped on a population panel of 80 human DNA samples representing four world populations (Additional file 2: DNA samples). The chromosome 3 insertion was determined to be human specific but fixed present in all individuals tested. A sequence alignment of these results for orangutan and human is shown in Figure 2. We could not identify any candidate loci in the chimpanzee genome. The closest match was only about $95 \%$ identical and did not share any of the Pan lineage-specific point mutations of the panTro2 Chr7 insertion (see Additional file 1: Figure S1).

Figure 2 shows that the Chr7 locus has a number of mutations different from the ancestral AluY consensus sequence that are shared in all the great ape species. These are highlighted in gray. Of the previously characterized Alu subfamilies, the Chr7 locus most closely matches the AluYe5 subfamily [22,23]. From this we can infer that the Chr7 locus was a member of the AluYe lineage upon its insertion. Figure 2 further illustrates that, post-insertion, lineage-specific point mutations have occurred at this locus in the various species over time. In the orangutan, the Chr7 Alu (hereafter designated as O:Chr7) left monomer has remained completely unscathed by about 16 million years of evolution and has no mutations compared to the ancestral AluY consensus sequence. The O:Chr7 right monomer independently acquired three sequential nucleotide substitutions (highlighted in yellow) which are also diagnostic mutations that define the young polymorphic AluYe5b5_Pongo subfamily. The first, at position 220, coincides with one of the diagnostic variants characteristic of the AluYe5 subfamily. Therefore it is possible that this is not a post-insertion orangutan-specific substitution, but rather was present upon insertion and later experienced a backward mutation in the common ancestor of gorilla, chimpanzee and human. Regardless, the other two of these three diagnostic substitutions in $\mathrm{O}$ : Chr7 are also present in all five of the youngest polymorphic AluYe5b5_Pongo elements in orangutan and are absent from the two fixed insertions. In gorilla, chimpanzee and human, the Chr7 insertion has acquired three different shared mutations (highlighted in green).
This alignment (Figure 2) provides strong evidence for the evolution of lineage-specific Alu insertion events from the ancestral Chr7 source element. There is further evidence that at least one of the orangutan-specific insertions, after acquiring two additional mutations (highlighted in aqua), remained active as a secondary source element generating new daughter copies.

These data provide strong evidence that the Chr7 ancestral $A l u$ insertion underwent a hierarchical accumulation of multiple post-insertion diagnostic substitutions in the orangutan, while also failing to accumulate the more likely random variants over the same evolutionary time period. This provides strong support that the Chr7 Alu insertion is the source element, or the original founder element, from which the younger, lineagespecific Alu insertions shown in Figure 2 derived. By counting the number of non-diagnostic random mutations that have occurred in each of the proposed offspring elements in the orangutan (accounting for $\mathrm{CpG}$ versus non-CpG) and using the calculated allele frequency distribution for each locus as determined by PCR (Tables 1 and 2 and Additional file 3: Table S2; Additional File 2, genotypes and allele frequencies), we constructed an estimated time-scale schematic for the propagation of this Alu lineage from the founder Chr7 locus (Figure 3). The evolutionary order in which each lineage-specific Alu insertion occurred is relatively straightforward along the majority of the estimated time-scale. However, it is not precisely clear which element represents the secondary source $A l u$ that sprouted the most recent Sumatran orangutan-specific subfamily shown in aqua. The O:Chr2b locus has the most nonCpG random mutations and the highest allele frequency (0.3513), indicating it is likely the oldest of the three insertions (Additional file 3: Table S2). However, this element integrated into the middle of an existing L1 element (Additional file 3) and presumably is unable to retrotranspose from this location. It is also exceedingly unlikely that all three loci accumulated the same two mutations independently post-insertion. Rather, it is much more likely that either one gave rise to the other two or, alternatively, the source of all three loci is no longer evident in the orangutan genome. Given that the $\mathrm{O}:$ Chr17 locus appears the most recent with a very low allele frequency (0.0811), then the O:Chr21 locus appears most likely to be the secondary source driver if we assume that one necessarily gave rise to the other 


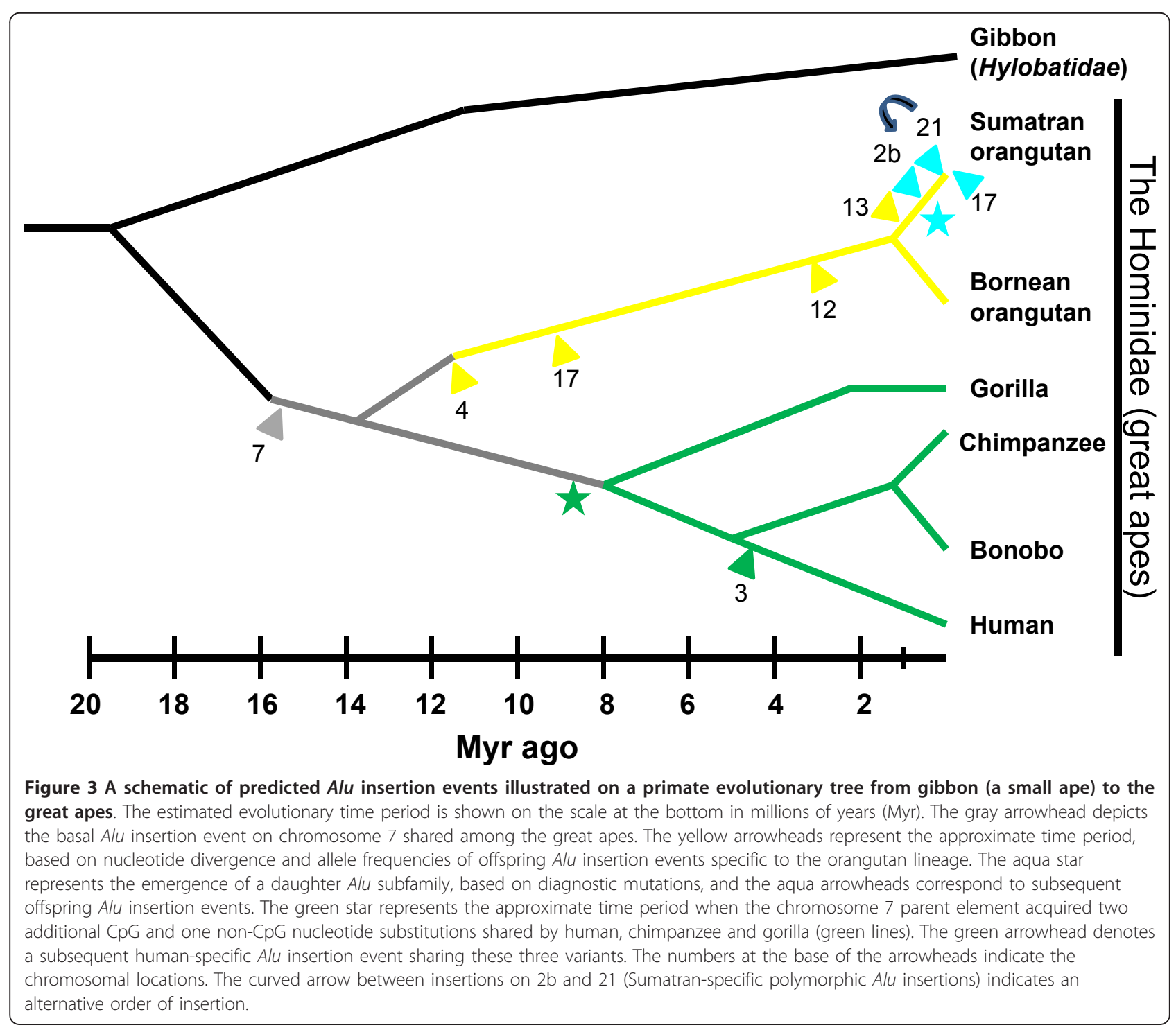

two. However, it seems more plausible that an unidentified secondary source driver produced all three copies and is simply not present in the ponAbe 2 assembly or has been lost from the genome due to lineage sorting. These alternative scenarios are depicted at the terminal branches of Figure 3.

\section{Discussion}

It is widely accepted that the expansion of Alu elements in primate genomes has occurred by using the L1 element enzymatic machinery for retrotransposition $[1,5]$. The identification of retrotranspositionally competent L1 elements is relatively straightforward as only fulllength elements having both open reading frames completely intact are capable of propagation via TPRT [24]. Only a limited number of L1 elements meet these criteria as the vast majority of L1s in primate genomes are truncated or have other disabling mutations [25]. The identification of potentially active Alu source elements is far more complicated because the majority of Alu elements are full-length and they do not contain a coding sequence. Recent research has investigated several structural features that influence the ability of $\mathrm{Alu}$ elements to replicate. These include the upstream flanking sequence, the integrity of the left monomer, the sequence identity to a known polymorphic subfamily, the distance of the Pol III termination signal from the 3' end of the element and the length and integrity of the poly(A) tail. A discussion of these factors supports the candidacy of our Chr7 Alu insertion as an ancestral source element.

The upstream flanking sequence of an Alu element has been reported to influence transcription ability [26-28]. The Chr7 Alu element reported here has what 
appears to be an intact TATA box (5'TATAAAAA3') cis regulatory transcription promoter immediately upstream to the $5^{\prime}$ TSD that is conserved in all species (Additional file 1: Figure S1). Although a TATA box is typically about 25 bp upstream of a transcription site and is usually the binding site for RNA polymerase II [29], TATA-box-like promoter sequences have been linked to the efficient transcription of the Alu-like human 7SL RNA gene by RNA polymerase III in vitro [30]. In addition, the presence of a 7SL sequence upstream has been shown to increase $A l u$ transcription [27]. However, RepeatMasker [31] analysis indicates that the upstream flanking sequence of this $A l u$ element is not a $7 \mathrm{SL}$ sequence but rather an ancient DNA transposon classified as a hAT-Charlie. Therefore, an alternative theory is that the 5'TATAAAAA3' sequence is not a functional TATA box but rather a simple variant of the classical TTTTAAAA or TTAAAA endonuclease cleavage site of L1 that is considered the preferred insertion site for Alu elements [32,33]. The potential role of this upstream sequence in the retrotransposition ability of this Alu element is not clear. However, the rhesus macaque genome (rheMac2) has a different sequence at this homologous position, 5'TATCAAAA3', and also does not have the Alu insertion.

Another factor determined to be critical for Alu replication is the structural integrity of the internal RNA Pol III promoter A and B boxes [4,7,34]. Two protein components of the signal recognition particle (SRP 9p and 14p) are believed to bind to specific $A l u$ sequences during L1-mediated TPRT [35] and these SRP9/14 binding sites in the left monomer are required for Alu activity $[4,7,34]$. Bennett and colleagues demonstrated experimentally that mutating the SRP9/14 binding site in the left monomer reduced $A l u$ mobilization efficiency to only $12 \%$ of normal, whereas a similar mutation in the right monomer, while also decreasing SRP9/14 binding, produced only a moderate decrease in retrotransposition efficiency, suggesting that an intact left monomer is more important for Alu mobilization [7]. The Chr7 Alu reported here has a completely conserved left monomer in orangutans, even though it is relatively old.

The degree of sequence variation between a candidate Alu 'master' element and a known polymorphic subfamily has also been reported to impact mobilization efficiency [7]. The O:Chr7 progenitor Alu element in the orangutan appears to have only two random substitutions that do not appear evident in its proposed progeny, and both variants are located in the right monomer. The first single nucleotide substitution is a CpG mutation at position 154 that is present in the ponAbe2 genome assembly (Figure 2) but does not completely segregate in all the orangutans we sequenced at this locus. Bornean orangutan KB5405 exhibited the original cytosine nucleotide at this position in all the clones we sequenced (Additional file 1: Figure S1). It is known that about $30 \%$ of all CpG sites reside within Alu elements [36] and that CpG sites have six to ten times faster mutation rates than non-CpG sites [37-39], increasing the potential for independently occurring random mutation events. The second single nucleotide substitution in O:Chr7 is a relatively recent $\mathrm{C}$ to $\mathrm{T}$ transition at position 247 that also does not completely segregate in all the orangutans we tested. It is completely absent from the Bornean orangutans (they all have the ancestral cytosine nucleotide) and remains polymorphic with an allele frequency of $50 \%$ in the tested Sumatran orangutans (Additional file 1: Figure S1). The overall lack of sequence divergence $(<1 \%)$ between the ancestral O:Chr7 Alu element and the consensus sequence of the young polymorphic AluYe5b5_Pongo subfamily in orangutan strongly supports its candidacy as the founder element from which the young subfamily derived.

The human Chr7 Alu element appears to have three substitutions that are not present in the $\mathrm{H}: \mathrm{Chr} 3 \mathrm{Alu}$ insertion, a CpG mutation at position 239 and two transversions: A to $\mathrm{T}$ at position 94 and $\mathrm{T}$ to $\mathrm{G}$ at position 173 (Figure 2). However, it is entirely possible, even probable, that all three substitutions occurred after the insertion in the $\mathrm{H}$ :Chr3 locus. The presence of a guanine residue at position 173 coincides with the consensus sequence of the human AluYf5 subfamily [31] and represents a single difference from the $A l u Y e 5$ subfamily consensus sequence $[22,23]$. Although the AluYf5 subfamily was likely mobilizing in primate genomes around the same time, based on the sequence structure of the locus it is unlikely that the human Chr7 Alu insertion contributed to the proliferation of this subfamily.

Another factor influencing Alu activity is the distance of the Pol III TTTT termination signal from the 3' end of the element. Comeaux and colleagues used Alu A tail constructs to experimentally determine the effect of various 3' end lengths on Alu mobilization [4]. They reported a strong decrease in Alu retrotransposition ability even with little sequence between the end of the A tail and the Pol III terminator. The Chr7 Alu element reported here has the Pol III transcription terminator (TTTT) in the 3' TSD immediately following the A tail, a characteristic associated with mobilization ability.

The length of the poly(A) tail has also been reported to influence $A l u$ retrotransposition activity, with longer A-tails free of nucleotide substitutions being more characteristic of young active source elements $[8,40]$. Mobilization ability in an ex vivo assay is reportedly very limited with a poly(A) tail less than 15 bp (base pairs) and increases thereafter to plateau at about $50 \mathrm{bp}$ [40]. Under endogenous conditions, there appears to be only 
a modest benefit to Alu retrotransposition efficiency once the $\operatorname{poly}(\mathrm{A})$ tail exceeds about $20 \mathrm{bp}$ [4]. The human Chr7 Alu element has a poly(A) tail length of 26 bp with two nucleotide substitutions and the O:Chr7 Alu element has a poly(A) tail length of $27 \mathrm{bp}$ with three nucleotide substitutions (Additional file 1: Figure S1). These poly(A) tail lengths are consistent with possible activity. In addition, the youngest orangutan Alu progeny element in this study (O:Chr17:56932716) displays a perfect 30 bp poly(A) tail (Additional file 3), consistent with the literature. Because older Alu elements tend to have less pristine poly(A) tails compared to younger elements, Comeaux and colleagues used $\mathrm{Alu}$ A tail constructs to experimentally determine the impact of A tail disruptions on retrotransposition efficiency [4]. They demonstrated that nucleotide disruptions within the poly $(\mathrm{A})$ tail are not created equal, in that adenine to thymine disruptions were relatively well tolerated with regard to maintaining the integrity of Alu mobilization, whereas nucleotide disruptions by cytosine or guanine resulted in greater impairment to retrotransposition efficiency [4]. The human Chr7 Alu element has a cytosine A tail disruption after 14 A-residues and a second one after 20 A-residues, perhaps impairing its current ability to propagate new copies. The O:Chr7 A tail has acquired a double cytosine (CC) mutation after only 10 A-residues and a third after only 16 A-residues (Additional file 1: Figure S1). These mutations may have rendered this ancestral Alu source element currently inactive.

With the exception of poly(A) tail disruptions, which may have occurred relatively recently, the ancestral Chr7 Alu insertion reported here possesses many of the classical hallmarks of being retrotranspositionally competent. Alu elements, like other retrotransposons, typically acquire nucleotide substitutions at a neutral rate after insertion [41]. Consequently, older elements tend to have a greater number of mutations (on average) than younger insertions. These acquired nucleotide substitutions often alter their ability to mobilize [4]. The Chr7 Alu reported here has remained highly conserved, especially in orangutans, even though it is approximately 16 million years old. This prompted us to speculate whether this element avoided the typical accumulation of random mutations because of its location in the $D G K B$ gene or simply by chance.

According to the UCSC Genome Browser $[18,19]$ Gene Sorter function, the human $D G K B$ gene is about $693 \mathrm{~kb}$ long (693,643 bp), of which only 2,415 bp is coding sequence $(<0.35 \%)$, comprising 804 amino acids distributed among 25 exons. Twenty-four introns make up the vast majority of the gene sequence. Zhang and colleagues [42] recently reported that, although Alu density is quite low in exons of genes (selected against), the Alu density in introns of genes is similar to the Alu density in intergenic regions of the genome, suggesting a similar selective pressure (essentially neutral). The $D G K B$ gene has no Alu element insertions within its exons or promoter regions, but has $113 \mathrm{Alu}$ element insertions within introns as identified by the TranspoGene database $[43,44]$. Of the $113 \mathrm{Alu}$ elements located within the gene, 19 were identified as $A l u \mathrm{Y}$ or younger, including the Alu element from this study, which is located in intron 20 of 24 . We screened the other 18 AluY elements to find those with the same species distribution to the $A l u$ element in this study and therefore expected to be of similar age. We selected seven full-length (> $275 \mathrm{bp)} A l u Y$ insertions from the DGKB gene that are shared in human, chimpanzee and orangutan, while absent from the rhesus macaque genome. We constructed a sequence alignment of these Alu elements from hg18 and ponAbe2 to compare their percent divergence from the consensus sequence compared to our element. The percent divergence of the human and orangutan AluY insertions was $6.1 \pm 1.4$ and $8.6 \pm 1.4$, respectively, compared to 3.6 and 3.3 respectively for the Alu element in this study (Additional file 4). Although this does not conclusively prove that the location of the Alu element in the DGKB gene has no effect, it does suggest that merely being present within a gene as opposed to within an intergenic sequence does not necessarily offer an $A l u$ element protection against ageassociated degradation. It also confirms that the Alu element in this study is unusually pristine for its age, a characteristic associated with mobilization ability. The reason for this, if not simply by chance alone, is not clear. The structure of this Alu element and its sequence evolution in multiple species is not consistent with a gene conversion event, nor is there any evidence of differential selection. It is possible that the Alu element is located in a more protected hypomethylated environment that is not similar to the other Alu element insertions we examined in the same gene, one of which was in the same intron. But to determine this would require a more comprehensive study of the $D G K B$ gene and its evolution.

We have estimated the Chr7 Alu insertion in this study to be about 16 million years old and concluded that this insertion was most likely a member of the AluYe lineage upon its insertion. In order to determine if this subfamily was actively mobilizing during the estimated time period, we examined data from a previous analysis of the AluYe lineage in which Salem and colleagues used PCR to determine the species distribution of 118 AluYe5 subfamily members [45]. Of these, about $32 \%$ (38 of 118) exhibited the same species distribution to the Alu element in this study while another $21 \%$ of the subfamily members (25 of 118) represented even 
older insertions that were also shared with siamang (present in human, chimpanzee, gorilla, orangutan and siamang). The remaining AluYe5 elements represented younger insertions, present in human, chimpanzee and gorilla but absent from orangutan (33\%), present in human and chimpanzee only (7\%) or were human-specific insertions (7\%). The findings of this previous study demonstrate that the Alu subfamily from which the Chr7 Alu insertion in this study is derived was actively propagating during the estimated time of its insertion. Moreover, in the orangutan lineage, the Chr7 ancestral Alu element underwent a hierarchical accumulation of multiple post-insertion diagnostic substitutions in the right arm, while also failing to accumulate the more likely random variants over the same evolutionary time period. It is inconceivable that by chance alone these post-insertion diagnostic substitutions just happen to match the young polymorphic AluYe5b5_Pongo elements in the orangutan, and that it is the only element identified in the orangutan genome to do so.

Our findings are consistent with a modified 'master gene' model of Alu amplification, or 'stealth model' for the expansion of lineage-specific Alu subfamilies [46]. It has been well established that $A l u$ subfamilies $>20$ million years old still have active members in primate genomes $[45,47]$. Studies of human Alu subfamilies have demonstrated that about $15 \%$ of subfamily members are active as secondary source elements [48], leading to a complex bush-like expansion of lineage-specific Alu subfamilies $[48,49]$. Under the stealth-driver model, an Alu lineage can remain quiescent for millions of years while maintaining low levels of retrotransposition activity to allow the lineage to persist over time $[46,50]$. In the case of the orangutan genome, the relative quiescence of Alu retrotransposition in the last several million years may have resulted from a population bottleneck or other demographic factors impacting their genomic architecture, and effectively disrupting the primary master-driver elements [51]. In this scenario, the survival of an Alu lineage would require the persistence of a few very old active copies that fortuitously avoided mutational decay, slowly giving rise to more recent active daughter elements. The recent expansion of the AluYe5b5_Pongo subfamily in orangutan is consistent with the existence of such a backseat driver. Conversely, in the human genome, the expansion of Alu from the Chr7 locus has remained quite limited, possibly due to the overall abundance of more robust Alu systems over the same evolutionary time period.

\section{Conclusions}

The objective of this study was to search for Alu source elements in the orangutan genome, against a background of relatively sparse young insertions. Using the youngest insertions as templates, we identified a much older, but nearly identical Alu insertion on Chr7. We demonstrated that this insertion is shared among great apes and possesses classical hallmarks of mobilization ability. We provided evidence for the concurrent evolution of lineage-specific insertion events from this source element in the orangutan and human genomes. Accumulated mutations within the poly (A) tails of these stealth drivers may have forced their eventual retirement. However, the sequential propagation of this Alu lineage in the orangutan genome, ongoing at a very low rate over millions of years of evolution and then recently sprouting young offspring, supports our finding of an ancient backseat driver of Alu element expansion.

\section{Methods}

\section{Orangutan-specific Alu subfamilies}

Of the orangutan-specific Alu subfamilies, three were determined to be the youngest based on their divergence from their respective consensus sequences. Using the standardized nomenclature for Alu repeats as a guideline [16], these three subfamilies have been termed AluYc1a5_Pongo, AluYe5a2_Pongo and AluYe5b5_Pongo. The consensus sequences are available as Additional file 5. The naming convention is as follows: The AluYc1a5_Pongo subfamily shares the same 12 bp deletion as the human AluYc and rhesus AluYRb lineages and likely derived from a common ancestor. In addition, it has six other mutations, only one of which is shared in the human and rhesus lineages, while the other five mutations seem unique to the orangutan lineage. The AluYe5a2_Pongo subfamily shares all the same insertions and deletions (indels) and substitutions of the human AluYe5 subfamily $[22,23]$, and has two additional transition mutations, hence 'a2'. Following this same naming convention, the AluYe5b5_Pongo subfamily also shares all the indels and substitutions of the human AluYe5 subfamily, but has five additional mutations, none of which are the same as the two characteristic of AluYe5a2_Pongo described above, hence 'b5'.

\section{Computational data collection}

We computationally searched the ponAbe2 (July 2007) orangutan genome assembly on the UCSC Genome Browser [18,19] using BLAT [17] for the closest matches to each of the three polymorphic Alu subfamily consensus sequences. Each locus was evaluated for species specificity using the UCSC Genome Browser and sequences were retrieved and aligned using BioEdit [52] or MegAlign with the ClustalW algorithm (DNASTAR, Inc. Version 5.0 for Windows).

\section{DNA}

The DNA panel used for PCR analysis of candidate Alu loci included human (Homo sapiens), bonobo 
chimpanzee (Pan paniscus), common chimpanzee (Pan troglodytes), lowland gorilla (Gorilla gorilla), Bornean orangutan (Pongo pygmaeus), Sumatran orangutan (Pongo abelii), siamang (Hylobates syndactylus), whitehanded gibbon (Hylobates lar), red-cheeked gibbon (Hylobates gabriellae), African green monkey (Chlorocebus aethiops) and rhesus macaque (Macaca mulatta). Orangutan DNA samples $(\mathrm{n}=37)$ were obtained from the Coriell Institute for Medical Research, Camden, NJ $(n=6)$, the San Diego Frozen Zoo $(n=19)$ and the National Institutes of Health National Cancer Institute $(\mathrm{n}=12)$. A list of these DNA samples is available in Additional file 2; DNA samples. The human-specific locus on chromosome 3 was analyzed in order to determine if it was polymorphic within a DNA panel of 80 individuals (20 African Americans, 20 Asians, 20 Europeans and 20 South Americans) obtained from the Coriell Institute for Medical Research, Camden, NJ (Additional file 2; DNA samples).

\section{PCR primer design}

Primers were manually designed in conserved regions of the orangutan, human, chimpanzee and rhesus macaque flanking sequences in order to increase the likelihood of successful amplification across various species under consideration of RepeatMasker output files [31]. For each locus, sequences were retrieved from UCSC $[18,19]$ and aligned using BioEdit [52] or MegAlign with the ClustalW algorithm. Each primer was checked with BLAT [17] to insure amplification of a single locus. In addition, a virtual PCR was performed for each locus using the in silico function of BLAT [17] in order to receive an estimated PCR product size for the empty (no insertion) and the filled size (insertion present). Primers were obtained from Sigma Aldrich (Woodlands, TX, USA).

\section{PCR analysis}

PCR amplifications were performed in $25 \mu \mathrm{L}$ reactions containing 15 to $50 \mathrm{ng}$ of template DNA, $200 \mathrm{nM}$ of each oligonucleotide primer, 1.5 to $3.0 \mathrm{mM} \mathrm{MgCl}_{2}, 10 \times$ PCR buffer (50 mM KCl, $10 \mathrm{mM}$ TrisHCl; pH 8.4), 0.2 $\mathrm{mM}$ deoxyribonucleotide triphosphates and 1 to $2 \mathrm{U}$ Taq DNA polymerase. PCR reactions were performed under the following conditions: initial denaturation at $94^{\circ} \mathrm{C}$ for 60 seconds, followed by 32 cycles of denaturation at $94^{\circ} \mathrm{C}$ for 30 seconds, 30 seconds at primer annealing temperature (Additional file 2; PCR primers and conditions), and extension at $72^{\circ} \mathrm{C}$ for 30 seconds. PCRs were terminated with a final extension at $72^{\circ} \mathrm{C}$ for 2 minutes. Fractionation of $20 \mu \mathrm{L}$ of each PCR product was performed in a horizontal gel chamber on a $2 \%$ agarose gel containing $0.2 \mu \mathrm{g} / \mathrm{mL}$ ethidium bromide for
60 to 70 minutes at $175 \mathrm{~V}$. UV-fluorescence was used to visualize the DNA fragments.

\section{Cloning and sequencing}

The original PCR primer pair used to screen the Chr7 Alu insertion resulted in a filled size of about $707 \mathrm{bp}$ (shown in Figure 1). In an effort to improve the efficiency of cloning and sequencing, a second set of PCR primers was designed to produce a smaller amplicon of about 475 bp (Additional file 2; PCR Primers). PCR fragments were gel purified using Wizard SV gel purification (Promega Corporation, Madison, WI, USA, cata$\log$ A9282) and cloned using TOPO TA cloning kits for sequencing (Invitrogen Corporation, Carlsbad, CA, USA, catalog K4575-40). A total of four to eight clones from each sample were sequenced using Sanger sequencing on an ABI 3130xl genome analyzer (Applied Biosystems, Inc., Foster City, CA, USA). Sequence quality was evaluated using ABI software Sequence Scanner v1.0. Sequences were analyzed using DNASTAR version 5.0 for windows and aligned using MegAlign. Sequence alignment figures were constructed by selecting the 'view alignment report' option in MegAlign, following manual formatting of 'alignment report contents' under Options. The output was saved as a text file, followed by manual refinement and labeling in Microsoft Word for windows.

\section{Description of additional data files}

The following additional data files are available with the online version of this paper. Additional file 1 is a sequence alignment report of the chromosome $7 \mathrm{Alu}$ insertion with the flanking sequence for multiple orangutan individuals and other primates obtained by Sanger sequencing of PCR amplicons. Additional file 2 is a series of tables listing the PCR primers and conditions, genotypes and allele frequencies, and DNA samples. Additional file 3 provides FASTA output for potential secondary source elements in orangutan. Additional file 4 is the TranspoGene database [44] output for the DGBK gene. Additional file 5 provides the Alu subfamily consensus sequences for the three youngest orangutanspecific subfamilies.

\section{Additional material}

Additional file 1: Figure S1 and Table S1. This file contains a sequence alignment report of the Chr7 Alu insertion with flanking sequence for multiple orangutan individuals and other primates obtained by Sanger sequencing of PCR amplicons and a list of the DNA samples used to construct the alignment.

Additional file 2: This file contains a series of tables listing the PCR primers and conditions, genotypes and allele frequencies, and DNA samples used in the study. 
Additional file 3: Table S2. This file provides FASTA output for three of the potential secondary source elements in the orangutan from the [ponAbe2] genome assembly and Table $\$ 2$.

Additional file 4: This file contains the TranspoGene database [42] output for the DGBK gene.

Additional file 5: This file provides the Alu subfamily consensus sequences for the three youngest orangutan-specific subfamilies.

\section{Abbreviations}

bp: base pair; DGKB: diacylglycerol kinase beta enzyme gene; Chr7: chromosome 7; kb: kilobase; LINE: long interspersed element; O:Chr: orangutan chromosome; PCR: polymerase chain reaction; Pol III: RNA polymerase III; SRP: signal recognition particle; TPRT: target-primed reverse transcription; TSD: target site duplication; UCSC: University of California Santa Cruz.

\section{Acknowledgements}

The authors would like to thank all the members of the Batzer Lab for their helpful suggestions and the Orangutan Genome Sequencing and Analysis Consortium. This research was supported by the National Institutes of Health R01 GM59290 (MAB). CPM was supported in part by the Louisiana Biomedical Research Network with funding from the National Center For Research Resources (Grant Number P20RR016456) and by the Louisiana Board of Regents Support Fund. The content is solely the responsibility of the authors and does not necessarily represent the official views of the National Center for Research Resources, the National Institutes of Health, or Louisiana Board of Regents.

\section{Author details}

${ }^{1}$ Department of Biological Sciences, Louisiana State University, 202 Life Sciences Building, Baton Rouge, LA 70803, USA. ²Department of Computer Science, Center for Computation and Technology (CCT), Louisiana State University, 316 Johnston Hall, Baton Rouge, LA 70803, USA. ${ }^{3}$ Department of Molecular and Cellular Physiology, Louisiana State University Health Sciences Center, 1501 Kings Highway, Shreveport, LA 71130, USA. ${ }^{4}$ School of Biological Sciences, Louisiana Tech University, Ruston, LA 71272, USA. ${ }^{5}$ Conservation and Research for Endangered Species (CRES), Zoological Society of San Diego, San Diego, CA 92112, USA. ${ }^{6}$ Institute for Systems Biology, Seattle, WA 98103, USA.

\section{Authors' contributions}

MKK, JAW and MAB designed the research and wrote the paper; MKK, JAW and CPM conducted the experiments; AFAS, RH, BU and MKK performed the repeat analysis of the ponAbe 2 genome assembly; OAR provided DNA samples and edited the manuscript. All authors read and approved the final manuscript.

\section{Authors' information}

CPM conducted experiments for this project in the Department of Biological Sciences, LSU-Baton Rouge as a participant in the Louisiana Biomedical Research Network (LBRN) while completing a degree in the School of Biological Sciences, Louisiana Tech University-Ruston, LA. CPM is currently a graduate student in the Department of Molecular and Cellular Physiology at LSUHSC-Shreveport.

\section{Competing interests}

The authors declare that they have no competing interests.

Received: 6 December 2011 Accepted: 30 April 2012

Published: 30 April 2012

\section{References}

1. Batzer MA, Deininger PL: Alu repeats and human genomic diversity. Nat Rev Genet 2002, 3:370-379.

2. Roy-Engel AM, Batzer MA, Deininger PL: Evolution of Human Retrosequences: Alu. Encyclopedia of Life Sciences (ELS). Chichester UK: John Wiley \& Sons, Ltd.; 2008.
3. Luan DD, Korman MH, Jakubczak JL, Eickbush TH: Reverse transcription of R2Bm RNA is primed by a nick at the chromosomal target site: a mechanism for non-LTR retrotransposition. Cell 1993, 72:595-605.

4. Comeaux MS, Roy-Engel AM, Hedges DJ, Deininger PL: Diverse cis factors controlling Alu retrotransposition: what causes Alu elements to die? Genome Res 2009, 19:545-555.

5. Dewannieux M, Esnault C, Heidmann T: LINE-mediated retrotransposition of marked Alu sequences. Nat Genet 2003, 35:41-48.

6. Ray DA, Xing J, Salem AH, Batzer MA: SINEs of a nearly perfect character. Syst Biol 2006, 55:928-935.

7. Bennett EA, Keller H, Mills RE, Schmidt S, Moran JV, Weichenrieder O, Devine SE: Active Alu retrotransposons in the human genome. Genome Res 2008, 18:1875-1883.

8. Roy-Engel AM, Salem AH, Oyeniran OO, Deininger L, Hedges DJ, Kilroy GE Batzer MA, Deininger PL: Active Alu element "A-tails": size does matter. Genome Res 2002, 12:1333-1344.

9. Locke DP, Hillier LW, Warren WC, Worley KC, Nazareth LV, Muzny DM, Yang SP, Wang Z, Chinwalla AT, Minx P, Mitreva M, Cook L, Delehaunty KD, Fronick C, Schmidt H, Fulton LA, Fulton RS, Nelson JO, Magrini V, Pohl C, Graves TA, Markovic C, Cree A, Dinh HH, Hume J, Kovar CL, Fowler GR, Lunter G, Meader S, Heger A, Ponting CP, Marques-Bonet T, Alkan C, Chen L, Cheng Z, Kidd JM, Eichler EE, White S, Searle S, Vilella AJ, Chen Y, Flicek P, Ma J, Raney B, Suh B, Burhans R, Herrero J, Haussler D, Faria R, Fernando O, Darré F, Farré D, Gazave E, Oliva M, Navarro A, Roberto R, Capozzi O, Archidiacono N, Della Valle G, Purgato S, Rocchi M, Konkel MK, Walker JA, Ullmer B, Batzer MA, Smit AF, Hubley R, Casola C, Schrider DR, Hahn MW, Quesada V, Puente XS, Ordoñez GR, López-Otín C, Vinar T, Brejova B, Ratan A, Harris RS, Miller W Kosiol C, Lawson HA, Taliwal V, Martins AL, Siepel A, Roychoudhury A, Ma X, Degenhardt J, Bustamante CD, Gutenkunst RN, Mailund T, Dutheil JY, Hobolth A, Schierup MH, Ryder OA, Yoshinaga Y, de Jong PJ, Weinstock GM, Rogers J, Mardis ER, Gibbs RA, Wilson RK: Comparative and demographic analysis of orang-utan genomes. Nature 2011, 469:529-533.

10. van Noordwijk MA, van Schaik CP: Development of ecological competence in Sumatran orangutans. Am J Phys Anthropol 2005, 127:79-94.

11. Chimpanzee Sequencing and Analysis Consortium: Initial sequence of the chimpanzee genome and comparison with the human genome. Nature 2005, 437:69-87.

12. Gibbs RA, Rogers J, Katze MG, Bumgarner R, Weinstock GM, Mardis ER, Remington KA, Strausberg RL, Venter JC, Wilson RK, Batzer MA, Bustamante CD, Eichler EE, Hahn MW, Hardison RC, Makova KD, Miller W, Milosavljevic A, Palermo RE, Siepel A, Sikela JM, Attaway T, Bell S, Bernard KE, Buhay CJ, Chandrabose MN, Dao M, Davis C, Delehaunty KD, Ding Y, Dinh HH, Dugan-Rocha S, Fulton LA, Gabisi RA, Garner T, Godfrey J, Hawes AC, Hernandez J, Hines S, Holder M, Hume J, Jhangiani SN, Joshi V, Khan ZM, Kirkness EF, Cree A, Fowler RG, Lee S, Lewis LR, Li Z, Liu YS, Moore SM, Muzny D, Nazareth LV, Ngo DN, Okwuonu GO, Pai G, Parker D, Paul HA, Pfannkoch C, Pohl CS, Rogers YH, Ruiz SJ, Sabo A, Santibanez J, Schneider BW, Smith SM, Sodergren E, Svatek AF, Utterback TR, Vattathil S, Warren W, White CS, Chinwalla AT, Feng Y, Halpern AL, Hillier LW, Huang X, Minx P, Nelson JO, Pepin KH, Qin X, Sutton GG, Venter E, Walenz BP, Wallis JW, Worley KC, Yang SP, Jones SM, Marra MA, Rocchi M, Schein JE, Baertsch R, Clarke L, Csürös M, Glasscock J, Harris RA, Havlak P, Jackson AR, Jiang H, Liu Y, Messina DN, Shen Y, Song HX, Wylie T, Zhang L, Birney E, Han K, Konkel MK, Lee J, Smit AF, Ullmer B, Wang H, Xing J, Burhans R, Cheng Z, Karro JE, Ma J, Raney B, She X, Cox MJ, Demuth JP, Dumas LJ, Han SG, Hopkins J, Karimpour-Fard A, Kim YH, Pollack JR, Vinar T, Addo-Quaye C, Degenhardt J, Denby A, Hubisz MJ, Indap A, Kosiol C, Lahn BT, Lawson HA, Marklein A, Nielsen R, Vallender EJ, Clark AG, Ferguson B, Hernandez RD, Hirani K, Kehrer-Sawatzki H, Kolb J, Patil S, Pu LL, Ren Y, Smith DG, Wheeler DA, Schenck I, Ball EV, Chen R, Cooper DN, Giardine B, Hsu F, Kent WJ, Lesk A, Nelson DL, O'brien WE, Prüfer K, Stenson PD, Wallace JC, Ke H, Liu XM, Wang P, Xiang AP, Yang F, Barber GP, Haussler D, Karolchik D, Kern AD, Kuhn RM, Smith KE, Zwieg AS: Evolutionary and biomedical insights from the rhesus macaque genome. Science 2007, 316:222-234.

13. Lander ES, Linton LM, Birren B, Nusbaum C, Zody MC, Baldwin J, Devon K, Dewar K, Doyle M, FitzHugh W, Funke R, Gage D, Harris K, Heaford A, Howland J, Kann L, Lehoczky J, LeVine R, McEwan P, McKernan K, Meldrim J, Mesirov JP, Miranda C, Morris W, Naylor J, Raymond C, Rosetti M, Santos R, Sheridan A, Sougnez C, Stange-Thomann N, Stojanovic N, Subramanian A, 
Wyman D, Rogers J, Sulston J, Ainscough R, Beck S, Bentley D, Burton J, Clee C, Carter N, Coulson A, Deadman R, Deloukas P, Dunham A, Dunham I, Durbin R, French L, Grafham D, Gregory S, Hubbard T, Humphray S, Hunt A, Jones M, Lloyd C, McMurray A, Matthews L, Mercer S, Milne S, Mullikin JC, Mungall A, Plumb R, Ross M, Shownkeen R, Sims S, Waterston RH, Wilson RK, Hillier LW, McPherson JD, Marra MA, Mardis ER, Fulton LA, Chinwalla AT, Pepin KH, Gish WR, Chissoe SL, Wendl MC, Delehaunty KD, Miner TL, Delehaunty A, Kramer JB, Cook LL, Fulton RS, Johnson DL, Minx PJ, Clifton SW, Hawkins T, Branscomb E, Predki P, Richardson P, Wenning S, Slezak T, Doggett N, Cheng JF, Olsen A, Lucas S, Elkin C, Uberbacher E, Frazier M, Gibbs RA, Muzny DM, Scherer SE, Bouck JB, Sodergren EJ, Worley KC, Rives CM, Gorrell JH, Metzker ML, Naylor SL, Kucherlapati RS, Nelson DL, Weinstock GM, Sakaki Y, Fujiyama A, Hattori M, Yada T, Toyoda A, Itoh T, Kawagoe C, Watanabe H, Totoki Y, Taylor T, Weissenbach J, Heilig R, Saurin W, Artiguenave F, Brottier P, Bruls T, Pelletier E, Robert C, Wincker P, Smith DR, Doucette-Stamm L, Rubenfield M, Weinstock K, Lee HM, Dubois J, Rosenthal A, Platzer M, Nyakatura G, Taudien S, Rump A, Yang H, Yu J, Wang J, Huang G, Gu J, Hood L, Rowen L, Madan A, Qin S, Davis RW, Federspiel NA, Abola AP, Proctor MJ, Myers RM, Schmutz J, Dickson M, Grimwood J, Cox DR, Olson MV, Kaul R, Raymond C, Shimizu N, Kawasaki K, Minoshima S, Evans GA, Athanasiou M, Schultz R, Roe BA, Chen F, Pan H, Ramser J, Lehrach H, Reinhardt R, McCombie WR, de la Bastide M, Dedhia N, Blöcker H, Hornischer K, Nordsiek G, Agarwala R, Aravind L, Bailey JA, Bateman A, Batzoglou S, Birney E, Bork P, Brown DG, Burge CB, Cerutti L, Chen HC, Church D, Clamp M, Copley RR, Doerks T, Eddy SR, Eichler EE, Furey TS, Galagan J, Gilbert JG, Harmon C, Hayashizaki Y, Haussler D, Hermjakob H, Hokamp K, Jang W, Johnson LS, Jones TA, Kasif S, Kaspryzk A, Kennedy S, Kent WJ, Kitts P, Koonin EV, Korf I, Kulp D, Lancet D, Lowe TM, McLysaght A, Mikkelsen T, Moran JV, Mulder N, Pollara VJ, Ponting CP, Schuler G, Schultz J, Slater G, Smit AF, Stupka E, Szustakowski J, Thierry-Mieg D, Thierry-Mieg J, Wagner L, Wallis J, Wheeler R, Williams A, Wolf YI, Wolfe KH, Yang SP, Yeh RF, Collins F, Guyer MS, Peterson J, Felsenfeld A, Wetterstrand KA, Patrinos A, Morgan MJ, de Jong P, Catanese JJ, Osoegawa K, Shizuya H, Choi S, Chen YJ, International Human Genome Sequencing Consortium: Initial sequencing and analysis of the human genome. Nature 2001, 409:860-921.

14. Hedges DJ, Callinan PA, Cordaux R, Xing J, Barnes E, Batzer MA: Differential alu mobilization and polymorphism among the human and chimpanzee lineages. Genome Res 2004, 14:1068-1075.

15. Liu GE, Alkan C, Jiang L, Zhao S, Eichler EE: Comparative analysis of Alu repeats in primate genomes. Genome Res 2009, 19:876-885.

16. Batzer MA, Deininger PL, Hellmann-Blumberg U, Jurka J, Labuda D, Rubin CM, Schmid CW, Zietkiewicz E, Zuckerkandl E: Standardized nomenclature for Alu repeats. J Mol Evol 1996, 42:3-6.

17. Kent WJ: BLAT-the BLAST-like alignment tool. Genome Res 2002, 12:656-664.

18. Kent WJ, Sugnet CW, Furey TS, Roskin KM, Pringle TH, Zahler AM, Haussler D: The human genome browser at UCSC. Genome Res 2002, 12:996-1006

19. UCSC Genome Browser. [http://genome.ucsc.edu].

20. Nagase T, Ishikawa K, Suyama M, Kikuno R, Miyajima N, Tanaka A, Kotani H, Nomura N, Ohara O: Prediction of the coding sequences of unidentified human genes. XI. The complete sequences of 100 new cDNA clones from brain which code for large proteins in vitro. DNA Res 1998, 5:277-286

21. Caricasole A, Bettini E, Sala C, Roncarati R, Kobayashi N, Caldara F, Goto K, Terstappen GC: Molecular cloning and characterization of the human diacylglycerol kinase beta (DGKbeta) gene: alternative splicing generates DGKbeta isotypes with different properties. J Biol Chem 2002, 277:4790-4796.

22. Jurka J: Repbase update: a database and an electronic journal of repetitive elements. Trends Genet 2000, 16:418-420.

23. Jurka J, Krnjajic M, Kapitonov W, Stenger JE, Kokhanyy O: Active Alu elements are passed primarily through paternal germlines. Theor Popul Biol 2002, 61:519-530.

24. Moran JV, Holmes SE, Naas TP, DeBerardinis RJ, Boeke JD, Kazazian HH Jr: High frequency retrotransposition in cultured mammalian cells. Cell 1996, 87:917-927.

25. Brouha B, Schustak J, Badge RM, Lutz-Prigge S, Farley AH, Moran JV, Kazazian HH Jr: Hot L1s account for the bulk of retrotransposition in the human population. Proc Natl Acad Sci USA 2003, 100:5280-5285.
26. Chesnokov I, Schmid CW: Flanking sequences of an Alu source stimulate transcription in vitro by interacting with sequence-specific transcription factors. J Mol Evol 1996, 42:30-36.

27. Roy AM, West NC, Rao A, Adhikari P, Aleman C, Barnes AP, Deininger PL: Upstream flanking sequences and transcription of SINEs. J Mol Biol 2000, 302:17-25.

28. Ullu E, Weiner AM: Upstream sequences modulate the internal promoter of the human 7SL RNA gene. Nature 1985, 318:371-374.

29. Smale ST, Kadonaga JT: The RNA polymerase II core promoter. Annu Rev Biochem 2003, 72:449-479.

30. Englert $M$, Felis $M$, Junker $V$, Beier $H$ : Novel upstream and intragenic control elements for the RNA polymerase III-dependent transcription of human 7SL RNA genes. Biochimie 2004, 86:867-874.

31. RepeatMasker Open-3.0. [http://www.repeatmasker.org].

32. Jurka J: Sequence patterns indicate an enzymatic involvement in integration of mammalian retroposons. Proc Natl Acad Sci USA 1997, 94:1872-1877.

33. Zhang K, Fan W, Deininger P, Edwards A, Xu Z, Zhu D: Breaking the computational barrier: a divide-conquer and aggregate based approach for Alu insertion site characterisation. Int I Comput Biol Drug Des 2009, 2:302-322.

34. Mills RE, Bennett EA, Iskow RC, Devine SE: Which transposable elements are active in the human genome? Trends Genet 2007, 23:183-191.

35. Boeke JD: LINEs and Alus-the polyA connection. Nat Genet 1997, 16:6-7.

36. Schmid CW: Human Alu subfamilies and their methylation revealed by blot hybridization. Nucleic Acids Res 1991, 19:5613-5617.

37. Batzer MA, Kilroy GE, Richard PE, Shaikh TH, Desselle TD, Hoppens $C L$, Deininger PL: Structure and variability of recently inserted Alu family members. Nucleic Acids Res 1990, 18:6793-6798.

38. Labuda D, Striker G: Sequence conservation in Alu evolution. Nucleic Acids Res 1989, 17:2477-2491.

39. Xing J, Hedges DJ, Han K, Wang H, Cordaux R, Batzer MA: Alu element mutation spectra: molecular clocks and the effect of DNA methylation. $J$ Mol Biol 2004, 344:675-682.

40. Dewannieux M, Heidmann T: Role of poly(A) tail length in Alu retrotransposition. Genomics 2005, 86:378-381.

41. Cordaux R, Lee J, Dinoso L, Batzer MA: Recently integrated Alu retrotransposons are essentially neutral residents of the human genome. Gene 2006, 373:138-144.

42. Zhang W, Edwards A, Fan W, Deininger P, Zhang K: Alu distribution and mutation types of cancer genes. BMC Genomics 2011, 12:157.

43. Levy A, Sela N, Ast G: TranspoGene and microTranspoGene: transposed elements influence on the transcriptome of seven vertebrates and invertebrates. Nucleic Acids Res 2008, 36:D47-D52.

44. TranspoGene Database. [http://transpogene.tau.ac.il].

45. Salem AH, Ray DA, Hedges DJ, Jurka J, Batzer MA: Analysis of the human Alu Ye lineage. BMC Evol Biol 2005, 5:18.

46. Han K, Xing J, Wang H, Hedges DJ, Garber RK, Cordaux R, Batzer MA: Under the genomic radar: the stealth model of Alu amplification. Genome Res 2005, 15:655-664.

47. Johanning K, Stevenson CA, Oyeniran OO, Gozal YM, Roy-Engel AM, Jurka J, Deininger PL: Potential for retroposition by old Alu subfamilies. J Mol Evol 2003, 56:658-664.

48. Cordaux R, Hedges DJ, Batzer MA: Retrotransposition of Alu elements: how many sources? Trends Genet 2004, 20:464-467.

49. Price AL, Eskin E, Pevzner PA: Whole-genome analysis of Alu repeat elements reveals complex evolutionary history. Genome Res 2004, 14:2245-2252.

50. Cordaux R, Batzer MA: The impact of retrotransposons on human genome evolution. Nat Rev Genet 2009, 10:691-703.

51. Hedges DJ, Batzer MA: From the margins of the genome: mobile elements shape primate evolution. BioEssays 2005, 27:785-794.

52. Hall TA: BioEdit: a user-friendly biological sequence alignment editor and analysis program for Windows 95/98/NT. Nucleic Acids Symp Ser 1999, 41:95-98.

doi:10.1186/1759-8753-3-8

Cite this article as: Walker et al:: Orangutan Alu quiescence reveals possible source element: support for ancient backseat drivers. Mobile DNA 2012 3:8. 